\title{
OPERATOR QUANTUM ERROR CORRECTION
}

\author{
DAVID W. KRIBS ${ }^{1,2}$, RAYMOND LAFLAMME $^{2,3}$, DAVID POULIN ${ }^{2,4}$, AND \\ MAIA LESOSKY ${ }^{1}$
}

\begin{abstract}
This paper is an expanded and more detailed version of the work [1] in which the Operator Quantum Error Correction protocol was introduced. This is a new scheme for the error correction of quantum operations that incorporates the known techniques - i.e. the standard error correction model, the method of decoherence-free subspaces, and the noiseless subsystem method as special cases, and relies on a generalized mathematical framework for noiseless subsystems that applies to arbitrary quantum operations. We also discuss a number of examples and introduce the notion of "unitarily noiseless subsystems".
\end{abstract}

A unified and generalized approach to quantum error correction, called Operator Quantum Error Correction (OQEC), was recently introduced in [1]. This model unifies all of the known techniques for the error correction of quantum operations - i.e. the standard model [2, 3, 4, 5], the method of decoherence-free subspaces [6, 7, ㅍ, 9] and the noiseless subsystem method [10, 11, 12] - under a single umbrella. An important new framework introduced as part of this scheme opens up the possibility of studying noiseless subsystems for arbitrary quantum operations.

This paper is an expanded and more detailed version of the work [1]. We provide complete details for proofs sketched there, and in some cases we present an alternative "operator" approach that leads to new information. In particular, we show that correction of the general codes introduced in [1] is equivalent to correction of certain operator algebras, and we use this to give a new proof for the main testable conditions in this scheme. In addition, we discuss a number of examples throughout the paper, and introduce the notion of "unitarily noiseless subsystems" (UNS) as a relaxation of the requirement in the noiseless subsystem formalism for immunity to errors.

We also connect this work with aspects of more recent OQEC related efforts. In particular, we show that the fundamental formula in the formulation of the "Quantum Computer Condition" recently introduced in [13] is captured as a special case of the UNS framework. 


\section{Preliminaries}

1.1. Quantum Operations. Let $\mathcal{H}$ be a (finite-dimensional) Hilbert space and let $\mathcal{B}(\mathcal{H})$ be the set of operators on $\mathcal{H}$. A quantum operation (or channel, or evolution) on $\mathcal{H}$ is a linear map $\mathcal{E}: \mathcal{B}(\mathcal{H}) \rightarrow \mathcal{B}(\mathcal{H})$ that is completely positive and preserves traces. Every channel has an operator-sum representation of the form $\mathcal{E}(\sigma)=\sum_{a} E_{a} \sigma E_{a}^{\dagger}, \forall \sigma \in$ $\mathcal{B}(\mathcal{H})$, where $\left\{E_{a}\right\} \subseteq \mathcal{B}(\mathcal{H})$ are the Kraus operators (or errors) associated with $\mathcal{E}$. As a convenience we shall write $\mathcal{E}=\left\{E_{a}\right\}$ when the $E_{a}$ determine $\mathcal{E}$ in this way.

The choice of operators that yield this form is not unique, but if $\mathcal{E}=\left\{E_{a}\right\}=\left\{F_{b}\right\}$ (without loss of generality assume the cardinalities of the sets are the same), then there is a scalar unitary matrix $U=\left(u_{a b}\right)$ such that $E_{a}=\sum_{b} u_{a b} F_{b} \forall a$. The map $\mathcal{E}$ is said to be unital or bistochastic if $\mathcal{E}(\mathbb{1})=\sum_{a} E_{a} E_{a}^{\dagger}=1$. Trace preservation of $\mathcal{E}$ can be phrased in terms of the error operators via the equation $\sum_{a} E_{a}^{\dagger} E_{a}=\mathbb{1}$, which is equivalent to the dual map for $\mathcal{E}$ being unital.

1.2. Standard Model for Quantum Error Correction. The "Standard Model" for the error correction of quantum operations [2, 3, 4, 5] consists of triples $(\mathcal{R}, \mathcal{E}, \mathcal{C})$ where $\mathcal{C}$ is a subspace, a quantum code, of a Hilbert space $\mathcal{H}$ associated with a given quantum system. The error $\mathcal{E}$ and recovery $\mathcal{R}$ are quantum operations on $\mathcal{B}(\mathcal{H})$ such that $\mathcal{R}$ undoes the effects of $\mathcal{E}$ on $\mathcal{C}$ in the following sense:

$$
(\mathcal{R} \circ \mathcal{E})(\sigma)=\sigma \quad \forall \sigma=P_{\mathcal{C}} \sigma P_{\mathcal{C}},
$$

where $P_{\mathcal{C}}$ is the projection of $\mathcal{H}$ onto $\mathcal{C}$.

When there exists such an $\mathcal{R}$ for a given pair $\mathcal{E}, \mathcal{C}$, the subspace $\mathcal{C}$ is said to be correctable for $\mathcal{E}$. The existence of a recovery operation $\mathcal{R}$ of $\mathcal{E}=\left\{E_{a}\right\}$ on $\mathcal{C}$ may be cleanly phrased in terms of the $\left\{E_{a}\right\}$ as follows [4, 5]:

$$
P_{\mathcal{C}} E_{a}^{\dagger} E_{b} P_{\mathcal{C}}=\lambda_{a b} P_{\mathcal{C}} \quad \forall a, b
$$

for some scalar matrix $\Lambda=\left(\lambda_{a b}\right)$. It is easy to see that this condition is independent of the operator-sum representation for $\mathcal{E}$.

1.3. Noiseless Subsystems and Decoherence-Free Subspaces. Let $\mathcal{E}=\left\{E_{a}\right\}$ be a quantum operation on $\mathcal{H}$. Let $\mathcal{A}$ be the $\mathrm{C}^{*}$-algebra generated by the $E_{a}$, so $\mathcal{A}=\operatorname{Alg}\left\{E_{a}, E_{a}^{\dagger}\right\}$. This is the set of polynomials in the $E_{a}$ and $E_{a}^{\dagger}$. As a $\dagger$-algebra (i.e., a finite-dimensional $\mathrm{C}^{*}$-algebra $[14,15,16]), \mathcal{A}$ has a unique decomposition up to unitary equivalence 
of the form

$$
\mathcal{A} \cong \bigoplus_{J}\left(\mathcal{M}_{m_{J}} \otimes \mathbb{1}_{n_{J}}\right)
$$

where $\mathcal{M}_{m_{J}}$ is the full matrix algebra $\mathcal{B}\left(\mathbb{C}^{m_{J}}\right)$ represented with respect to a given orthonormal basis and $\mathbb{1}_{n_{J}}$ is the identity on $\mathbb{C}^{n_{J}}$. This means there is an orthonormal basis such that the matrix representations of operators in $\mathcal{A}$ with respect to this basis have the form in Eq. (3). Typically $\mathcal{A}$ is called the interaction algebra associated with the operation $\mathcal{E}$.

The standard "noiseless subsystem" method of quantum error correction [10, 11, 12] makes use of the operator algebra structure of the noise commutant associated with $\mathcal{E}$;

$$
\mathcal{A}^{\prime}=\left\{\sigma \in \mathcal{B}(\mathcal{H}): E \sigma=\sigma E \forall E \in\left\{E_{a}, E_{a}^{\dagger}\right\}\right\}
$$

Observe that when $\mathcal{E}$ is unital, all the states encoded in $\mathcal{A}^{\prime}$ are immune to the errors of $\mathcal{E}$. Thus, this is in effect a method of passive error correction. The structure of $\mathcal{A}$ given in Eq. (3) implies that the noise commutant is unitarily equivalent to

$$
\mathcal{A}^{\prime} \cong \bigoplus_{J}\left(\mathbb{1}_{m_{J}} \otimes \mathcal{M}_{n_{J}}\right)
$$

It is obvious from Eqs. (34) that elements of $\mathcal{A}^{\prime}$ are immune to the errors of $\mathcal{A}$ when $\mathcal{E}$ is unital. In [17] the converse of this statement was proved. Specifically, when $\mathcal{E}$ is unital the noise commutant coincides with the fixed point set for $\mathcal{E}$; i.e.,

$$
\mathcal{A}^{\prime}=\operatorname{Fix}(\mathcal{E})=\left\{\sigma \in \mathcal{B}(\mathcal{H}): \mathcal{E}(\sigma)=\sum_{a} E_{a} \sigma E_{a}^{\dagger}=\sigma\right\}
$$

This is precisely the reason that $\mathcal{A}^{\prime}$ may be used to produce noiseless subsystems for unital $\mathcal{E}$. We note that the noiseless subsystem method may be regarded as containing the method of decoherence-free subspaces [6, 7, 8, 9] as a special case, in the sense that this method makes use of the "unampliated" summands, $\mathbb{1}_{m_{J}} \otimes \mathcal{M}_{n_{J}}$ where $m_{J}=1$, inside the noise commutant $\mathcal{A}^{\prime}$ for encoding information.

While many physical noise models satisfy the unital constraint, the generic quantum operation is non-unital. Below we show how shifting the focus from $\mathcal{A}^{\prime}$ to $\operatorname{Fix}(\mathcal{E})$ (and related sets) quite naturally leads to the notion of noiseless subsystems that applies to arbitrary quantum operations. 


\section{Noiseless Subsystems For Arbitrary Quantum OPERATIONS}

In this section we describe a generalized mathematical framework for noiseless subsystems that applies to arbitrary (not necessarily unital) quantum operations and serves as a building block for the OQEC scheme presented below. Previous discussions of noiseless subsystems were not always restricted to unital maps [10, 11, 12]. However, the standard mathematical framework either explicitly focuses on unital maps, or does so implicitly by relying on the algebraic approach outlined above. The results presented in [12] apply to an arbitrary map in the Markovian approximation, and also yield an algebraic structure.

Note that the structure of the algebra $\mathcal{A}$ given in Eq. (3) induces a natural decomposition of the Hilbert space

$$
\mathcal{H}=\bigoplus_{J} \mathcal{H}_{J}^{A} \otimes \mathcal{H}_{J}^{B}
$$

where the "noisy subsystems" $\mathcal{H}_{J}^{A}$ have dimension $m_{J}$ and the "noiseless subsystems" $\mathcal{H}_{J}^{B}$ have dimension $n_{J}$. For brevity, we focus on the case where information is encoded in a single noiseless sector of $\mathcal{B}(\mathcal{H})$, and hence

$$
\mathcal{H}=\left(\mathcal{H}^{A} \otimes \mathcal{H}^{B}\right) \oplus \mathcal{K}
$$

with $\operatorname{dim}\left(\mathcal{H}^{A}\right)=m, \operatorname{dim}\left(\mathcal{H}^{B}\right)=n$ and $\operatorname{dim} \mathcal{K}=\operatorname{dim} \mathcal{H}-m n$. We shall write $\sigma^{A}$ for operators in $\mathcal{B}\left(\mathcal{H}^{A}\right)$ and $\sigma^{B}$ for operators in $\mathcal{B}\left(\mathcal{H}^{B}\right)$. Thus the restriction of the noise commutant $\mathcal{A}^{\prime}$ to $\mathcal{H}^{A} \otimes \mathcal{H}^{B}$ consists of the operators of the form $\sigma=\mathbb{1}^{A} \otimes \sigma^{B}$ where $\mathbb{1}^{A}$ is the identity element of $\mathcal{B}\left(\mathcal{H}^{A}\right)$.

For notational purposes, assume that ordered orthonormal bases have been chosen for $\mathcal{H}^{A}=\operatorname{span}\left\{\left|\alpha_{i}\right\rangle\right\}_{i=1}^{m}$ and $\mathcal{H}^{B}=\operatorname{span}\left\{\left|\beta_{k}\right\rangle\right\}_{k=1}^{n}$ that yield the matrix representation of the corresponding subalgebra of $\mathcal{A}^{\prime}$ as $\mathbb{1}^{A} \otimes \mathcal{B}\left(\mathcal{H}^{B}\right) \cong \mathbb{1}_{m} \otimes \mathcal{M}_{n}$. We let

$$
P_{k l} \equiv\left|\alpha_{k}\right\rangle\left\langle\alpha_{l}\right| \otimes \mathbb{1}^{B} \quad \forall 1 \leq k, l \leq m
$$

denote the corresponding family of "matrix units" in $\mathcal{A}$ associated with this decomposition. The following identities are readily verified and are the defining properties for a family of matrix units:

$$
\begin{aligned}
P_{k l} & =P_{k k} P_{k l} P_{l l} \quad \forall 1 \leq k, l \leq m \\
P_{k l}^{\dagger} & =P_{l k} \quad \forall 1 \leq k, l \leq m \\
P_{k l} P_{l^{\prime} k^{\prime}} & =\left\{\begin{array}{cl}
P_{k k^{\prime}} & \text { if } l=l^{\prime} \\
0 & \text { if } l \neq l^{\prime}
\end{array}\right.
\end{aligned}
$$

With these properties in hand, the following result is readily proved. 
Lemma 2.1. The map $\Gamma: \mathcal{B}(\mathcal{H}) \rightarrow \mathcal{B}(\mathcal{H})$ given by $\Gamma=\left\{P_{k l}\right\}$ satisfies the following:

$$
\Gamma(\sigma)=\sum_{k, l} P_{k l} \sigma P_{k l}^{\dagger}=\mathbb{1}^{A} \otimes \operatorname{Tr}_{A}(\sigma)
$$

for all operators $\sigma \in \mathcal{B}(\mathcal{H})$, so in particular $\Gamma\left(\sigma^{A} \otimes \sigma^{B}\right) \propto \mathbb{1}^{A} \otimes \sigma^{B}$ for all $\sigma^{A}$ and $\sigma^{B}$.

Note 2.2. While we have stated this result as part of a discussion on a subalgebra of a noise commutant, it is valid for any $\dagger$-algebra $\mathfrak{B} \cong$ $\mathbb{1}^{A} \otimes \mathcal{B}\left(\mathcal{H}^{B}\right)$ with matrix units $\left\{P_{k l}\right\}$ generating the algebra $\mathcal{B}\left(\mathcal{H}^{A}\right) \otimes \mathbb{1}^{B}$.

We now turn to the generalized noiseless subsystems method. In this framework, the quantum information is encoded in $\sigma^{B}$; i.e., the state of the noiseless subsystem. But it is not necessary for the noisy subsystem to remain in the maximally mixed state $\mathbb{1}^{A}$ under $\mathcal{E}$, it could in principle get mapped to any other state.

In order to formalize this idea, define for a fixed decomposition $\mathcal{H}=$ $\left(\mathcal{H}^{A} \otimes \mathcal{H}^{B}\right) \oplus \mathcal{K}$ the set of operators

$$
\mathfrak{A}=\left\{\sigma \in \mathcal{B}(\mathcal{H}): \sigma=\sigma^{A} \otimes \sigma^{B}, \text { for some } \sigma^{A} \text { and } \sigma^{B}\right\} .
$$

Notice that this set has the structure of a semigroup and includes operator algebras such as $\mathfrak{A}_{0} \equiv \mathbb{1}^{A} \otimes \mathcal{B}\left(\mathcal{H}^{B}\right)$ and $\left|\alpha_{k}\right\rangle\left\langle\alpha_{k}\right| \otimes \mathcal{B}\left(\mathcal{H}^{B}\right)$. Define the projection $P_{\mathfrak{A}} \equiv P_{11}+\ldots+P_{m m}$, so that $P_{\mathfrak{A}} \mathcal{H}=\mathcal{H}^{A} \otimes \mathcal{H}^{B}$, $P_{\mathfrak{A}}^{\perp}=\mathbb{1}-P_{\mathfrak{A}}$ and $P_{\mathfrak{A}}^{\perp} \mathcal{H}=\mathcal{K}$. We also define a superoperator $\mathcal{P}_{\mathfrak{A}}$ by the action $\mathcal{P}_{\mathfrak{A}}(\cdot)=P_{\mathfrak{A}}(\cdot) P_{\mathfrak{A}}$.

Lemma 2.3. Given a fixed decomposition $\mathcal{H}=\left(\mathcal{H}^{A} \otimes \mathcal{H}^{B}\right) \oplus \mathcal{K}$ and a quantum operation $\mathcal{E}$ on $\mathcal{B}(\mathcal{H})$, the following four conditions are equivalent, and are the defining properties of the noiseless subsystem $B$ :

(1) $\forall \sigma^{A} \forall \sigma^{B}, \exists \tau^{A}: \mathcal{E}\left(\sigma^{A} \otimes \sigma^{B}\right)=\tau^{A} \otimes \sigma^{B}$

(2) $\forall \sigma^{B}, \exists \tau^{A}: \mathcal{E}\left(\mathbb{1}^{A} \otimes \sigma^{B}\right)=\tau^{A} \otimes \sigma^{B}$

(3) $\forall \sigma \in \mathfrak{A}:\left(\operatorname{Tr}_{A} \circ \mathcal{P}_{\mathfrak{A}} \circ \mathcal{E}\right)(\sigma)=\operatorname{Tr}_{A}(\sigma)$.

Proof. The implications 1. $\Rightarrow$ 2. and 1. $\Rightarrow$ 3. are trivial. To prove 2 . $\Rightarrow 1$., first let $|\psi\rangle \in \mathcal{H}^{B}$ and put $P=|\psi\rangle\langle\psi|$. Suppose that $\left\{\left|\alpha_{k}\right\rangle\right\}$ is an orthonormal basis for $\mathcal{H}^{A}$. Then $\sum_{k=1}^{m}\left|\alpha_{k}\right\rangle\left\langle\alpha_{k}\right|=\mathbb{1}^{A}$ and by 2 . and the positivity of $\mathcal{E}$ we have for all $k$,

$$
\begin{aligned}
0 \leq \mathcal{E}\left(\left|\alpha_{k}\right\rangle\left\langle\alpha_{k}\right| \otimes P\right) & \leq \mathcal{E}\left(\mathbb{1}^{A} \otimes P\right) \\
& =\tau^{A} \otimes P \\
& =\left(\mathbb{1}^{A} \otimes P\right)\left(\tau^{A} \otimes P\right)\left(\mathbb{1}^{A} \otimes P\right) .
\end{aligned}
$$

It follows that there are positive operators $\sigma_{\psi, k} \in \mathcal{B}\left(\mathcal{H}^{A}\right)$ such that $\mathcal{E}\left(\left|\alpha_{k}\right\rangle\left\langle\alpha_{k}\right| \otimes P\right)=\sigma_{\psi, k} \otimes P$ for all $k$. 
In fact, the operators $\sigma_{\psi, k}$ do not depend on $|\psi\rangle$. To verify this claim, for clarity we shall suppose that $\operatorname{dim} \mathcal{H}^{B}=2$. The case of general $\mathcal{H}^{B}$ easily follows. So let $\left|\psi_{i}\right\rangle, i=1,2$, be an orthonormal basis for $\mathcal{H}^{B}$. Let $P_{i}=\left|\psi_{i}\right\rangle\left\langle\psi_{i}\right|, i=1,2$, and put $P_{ \pm}=| \pm\rangle\langle \pm|$where $| \pm\rangle=\frac{1}{\sqrt{2}}\left(\left|\psi_{1}\right\rangle \pm\left|\psi_{2}\right\rangle\right)$. Fix $\alpha=\alpha_{k}$. By the above argument, there are operators $\sigma_{ \pm, \alpha}$ and $\sigma_{i, \alpha}$ on $\mathcal{H}^{A}$ such that

$$
\mathcal{E}\left(|\alpha\rangle\langle\alpha| \otimes P_{ \pm}\right)=\sigma_{ \pm, \alpha} \otimes P_{ \pm} \quad \text { and } \quad \mathcal{E}\left(|\alpha\rangle\langle\alpha| \otimes P_{i}\right)=\sigma_{i, \alpha} \otimes P_{i}
$$

In particular, as $\mathbb{1}^{B}=P_{+}+P_{-}=P_{1}+P_{2}$, we have

$$
\begin{aligned}
\mathcal{E}\left(|\alpha\rangle\langle\alpha| \otimes \mathbb{1}^{B}\right) & =\sigma_{1, \alpha} \otimes P_{1}+\sigma_{2, \alpha} \otimes P_{2} \\
& =\sigma_{+, \alpha} \otimes P_{+}+\sigma_{-, \alpha} \otimes P_{-} .
\end{aligned}
$$

If we compress this equation by the projection $\mathbb{1}^{A} \otimes P_{1}$, we obtain

$$
\begin{aligned}
\left(\mathbb{1}^{A} \otimes P_{1}\right) \mathcal{E}\left(|\alpha\rangle\langle\alpha| \otimes \mathbb{1}^{B}\right)\left(\mathbb{1}^{A} \otimes P_{1}\right) & =\sigma_{1, \alpha} \otimes P_{1} \\
& =\frac{1}{2}\left(\sigma_{+, \alpha}+\sigma_{-, \alpha}\right) \otimes P_{1} .
\end{aligned}
$$

Thus, $\sigma_{1, \alpha}=\frac{1}{2}\left(\sigma_{+, \alpha}+\sigma_{-, \alpha}\right)$ and since the same identity holds for $\sigma_{2, \alpha}$ when we compress by $\mathbb{1}^{A} \otimes P_{2}$, we obtain $\sigma_{1, \alpha}=\sigma_{2, \alpha}$. As $|\alpha\rangle$ and $\left|\psi_{i}\right\rangle$, $i=1,2$, were chosen arbitrarily, the claim holds. Condition 1 . now follows from the linearity of $\mathcal{E}$.

To prove 3. $\Rightarrow$ 2., first note that since $\mathcal{E}$ and $\operatorname{Tr}_{A}$ are positive and trace preserving, 3. implies that $\left(\mathcal{P}_{\mathfrak{A}} \circ \mathcal{E}\right)(\sigma)=\mathcal{E}(\sigma)$ for all $\sigma \in \mathfrak{A}$. Now fix $|\psi\rangle \in \mathcal{H}^{B}$ and put $\sigma=\mathbb{1}^{A} \otimes P$ where $P=|\psi\rangle\langle\psi|$. Then by 3. we have

$$
\operatorname{Tr}_{A}\left(\left(\mathbb{1}^{A} \otimes P\right) \mathcal{E}(\sigma)\left(\mathbb{1}^{A} \otimes P\right)\right)=\operatorname{Tr}_{A}(\sigma)
$$

It follows again from the trace preservation and positivity of $\operatorname{Tr}_{A}$ and $\mathcal{E}$ that $\sigma \mathcal{E}(\sigma) \sigma=\mathcal{E}(\sigma)$, and hence there is a $\tau^{A}$ such that $\mathcal{E}(\sigma)=\tau^{A} \otimes P$. The above argument may now be used to show that $\tau^{A}$ is independent of $|\psi\rangle$, and the rest follows from the linearity of $\mathcal{E}$.

Definition 2.4. The subsystem $B$ is said to be noiseless for $\mathcal{E}$ when it satisfies one - and hence all — of the conditions in Lemma 2.3.

Note that the generalized definition of noiseless subsystems coincides with the standard definition when $\operatorname{dim}\left(\mathcal{H}^{A}\right)=1$. Hence, the notion of decoherence-free subspaces is not altered by this generalization. We next give necessary and sufficient conditions for a subsystem to be noiseless for a map $\mathcal{E}=\left\{E_{a}\right\}$.

Theorem 2.5. Let $\mathcal{E}=\left\{E_{a}\right\}$ be a quantum operation on $\mathcal{B}(\mathcal{H})$ and let $\mathfrak{A}$ be a semigroup in $\mathcal{B}(\mathcal{H})$ as above. Then the following three conditions are equivalent: 
(1) The B-sector of $\mathfrak{A}$ encodes a noiseless subsystem for $\mathcal{E}$ (decoherencefree subspace in the case $m=1$ ), as in Definition 2.4.

(2) The subspace $P_{\mathfrak{A}} \mathcal{H}=\mathcal{H}^{A} \otimes \mathcal{H}^{B}$ is invariant for the operators $E_{a}$ and the restrictions $\left.E_{a}\right|_{P_{\mathfrak{A}} \mathcal{H}}$ belong to the algebra $\mathcal{B}\left(\mathcal{H}^{A}\right) \otimes \mathbb{1}^{B}$.

(3) The following two conditions hold:

$$
P_{k k} E_{a} P_{l l}=\lambda_{a k l} P_{k l} \quad \forall a, k, l
$$

for some set of scalars $\left(\lambda_{a k l}\right)$ and

$$
E_{a} P_{\mathfrak{A}}=P_{\mathfrak{A}} E_{a} P_{\mathfrak{A}} \quad \forall a .
$$

Proof. Since the matrix units $\left\{P_{k l}\right\}$ generate $\mathcal{B}\left(\mathcal{H}^{A}\right) \otimes \mathbb{1}^{B}$ as an algebra, it follows that 3. is a restatement of 2. To prove the necessity of Eqs. (910) for 1., let $\Gamma: \mathcal{B}(\mathcal{H}) \rightarrow \mathbb{1}^{A} \otimes \mathcal{B}\left(\mathcal{H}^{B}\right)$ be defined by the matrix units for $\mathfrak{A}$ as above and note that Lemma 2.1 and Lemma 2.3 imply

$$
(\Gamma \circ \mathcal{E} \circ \Gamma)(\sigma) \propto \Gamma(\sigma) \text { for all } \sigma \in \mathcal{B}(\mathcal{H})
$$

As in the proof of Lemma 2.3, the proportionality factor cannot depend on $\sigma$, so the sets of operators $\left\{P_{k i} E_{a} P_{j l}\right\}$ and $\left\{\lambda P_{k^{\prime} l^{\prime}}\right\}$ define the same map for some scalar $\lambda$. We may thus find a set of scalars $\mu_{k i a j l, k^{\prime} l^{\prime}}$ such that

$$
P_{k i} E_{a} P_{j l}=\sum_{k^{\prime} l^{\prime}} \mu_{k i a j l, k^{\prime} l^{\prime}} P_{k^{\prime} l^{\prime}}
$$

Multiplying both sides of this equality on the right by $P_{l}$ and on the left by $P_{k}$, we see that $\mu_{k i a j l, k^{\prime} l^{\prime}}=0$ when $k \neq k^{\prime}$ or $l \neq l^{\prime}$. This implies Eq. (9) with $\lambda_{a k l}=\mu_{k k a l l, k l}$.

For the second condition, note that as a consequence of Lemma 2.3. we have $P_{\mathfrak{A}}^{\perp} \mathcal{E}\left(P_{\mathfrak{A}}(\sigma)\right) P_{\mathfrak{A}}^{\perp}=0$ for all $\sigma \in \mathcal{B}(\mathcal{H})$. Equation (10) follows from this observation via consideration of the operator-sum representation for $\mathcal{E}$.

To prove sufficiency of Eqs. (91), (10) for 1., we use the identity $P_{\mathfrak{A}}=\sum_{k=1}^{m} P_{k}$ to establish for all $\sigma=P_{\mathfrak{A}} \sigma \in \mathfrak{A}$,

$$
\begin{aligned}
\mathcal{E}(\sigma) & =\left(P_{\mathfrak{A}}+P_{\mathfrak{A}}^{\perp}\right) \sum_{a} E_{a} \sigma E_{a}^{\dagger}\left(P_{\mathfrak{A}}+P_{\mathfrak{A}}^{\perp}\right) \\
& =\sum_{a} P_{\mathfrak{A}} E_{a} \sigma E_{a}^{\dagger} P_{\mathfrak{A}} \\
& =\sum_{a, k, k^{\prime}} P_{k k} E_{a} \sigma E_{a}^{\dagger} P_{k^{\prime} k^{\prime}}
\end{aligned}
$$


Combining this with the identity

$$
\sigma^{A} \otimes \sigma^{B}=P_{\mathfrak{A}}\left(\sigma^{A} \otimes \sigma^{B}\right) P_{\mathfrak{A}}=\sum_{l, l^{\prime}} P_{l l}\left(\sigma^{A} \otimes \sigma^{B}\right) P_{l^{\prime} l^{\prime}}
$$

implies for all $\sigma=\sigma^{A} \otimes \sigma^{B} \in \mathfrak{A}$,

$$
\begin{aligned}
\mathcal{E}\left(\sigma^{A} \otimes \sigma^{B}\right) & =\sum_{a, k, k^{\prime}, l, l^{\prime}} P_{k k} E_{a} P_{l l}\left(\sigma^{A} \otimes \sigma^{B}\right) P_{l^{\prime} l^{\prime}} E_{a}^{\dagger} P_{k^{\prime} k^{\prime}} \\
& =\sum_{a, k, k^{\prime}, l, l^{\prime}} \lambda_{a k l} \bar{\lambda}_{a k^{\prime} l^{\prime}} P_{k l}\left(\sigma^{A} \otimes \sigma^{B}\right) P_{l^{\prime} k^{\prime}}
\end{aligned}
$$

The proof now follows from the fact that the matrix units $P_{k l}$ act trivially on the $\mathcal{B}\left(\mathcal{H}^{B}\right)$ sector.

Remark 2.6. In the case that the semigroup $\mathfrak{A}$ is determined by a matrix block inside the noise commutant $\mathcal{A}^{\prime}$ for a unital channel $\mathcal{E}=\left\{E_{a}\right\}$, and hence arises through the algebraic approach as in the discussion at the start of this section, the conditions Eqs. (910) follow from the structure of $\mathcal{A}=\operatorname{Alg}\left\{E_{a}, E_{a}^{\dagger}\right\}$ determined by the matrix units $P_{k l}$. However, Eqs. (910) do not necessarily imply that the noiseless operators of $\mathfrak{A}$ are in the commutant of the interaction algebra $\mathcal{A}$. In fact, recent work [18] gives a method to find all noiseless subsystems for arbitrary quantum operations. It is shown that the noise commutant still yields noiseless subsystems (even in the non-unital case), and that all other noiseless subsystems are shown to arise through an interplay between the noise commutant and projections $P$ that satisfy the equation $\mathcal{E}(P)=P \mathcal{E}(P) P$.

Example 2.7. As a simple illustration of a noiseless subsystem in a non-unital case, consider the quantum channel $\mathcal{E}: \mathcal{M}_{4} \rightarrow \mathcal{M}_{4}$ with errors $\mathcal{E}=\left\{E_{1}, E_{2}\right\}$ obtained as follows. Fix $\gamma, 0 \leq \gamma \leq 1$, and with respect to the basis $\{|0\rangle,|1\rangle\}$ let

$$
F_{0}=\left(\begin{array}{cc}
\sqrt{\gamma} & 0 \\
0 & \sqrt{1-\gamma}
\end{array}\right) \quad \text { and } \quad F_{1}=\left(\begin{array}{cc}
0 & \sqrt{\gamma} \\
\sqrt{1-\gamma} & 0
\end{array}\right)
$$

Then define $E_{i}=F_{i} \otimes \mathbb{1}_{2}$, for $i=0,1$. That $\sum_{i} E_{i}^{\dagger} E_{i}=\mathbb{1}_{4}$ follows from $\sum_{i} F_{i}^{\dagger} F_{i}=\mathbb{1}_{2}$, which can be verified straightforwardly.

Decompose $\mathbb{C}^{4}=\mathcal{H}^{A} \otimes \mathcal{H}^{B}$ with respect to the standard basis, so that $\mathcal{H}^{A}=\mathcal{H}^{B}=\mathbb{C}^{2}$. Then for all $\sigma=\sigma^{A} \otimes \sigma^{B}$, we have

$$
\mathcal{E}(\sigma)=\sum_{i=0}^{1} E_{i}\left(\sigma^{A} \otimes \sigma^{B}\right) E_{i}^{\dagger}=\left(\sum_{i=0}^{1} F_{i} \sigma^{A} F_{i}^{\dagger}\right) \otimes \sigma^{B} .
$$


With the operator $\tau^{A}$ from Lemma 2.3 given by $\tau^{A}=\sum_{i} F_{i} \sigma^{A} F_{i}^{\dagger}$. It follows that $\mathcal{H}^{B}$ encodes a noiseless subsystem for $\mathcal{E}$. Also observe that, as opposed to the completely error-free evolution that characterizes the unital case, in this case we have $\mathcal{E}\left(\mathbb{1}^{A} \otimes \sigma^{B}\right) \neq \mathbb{1}^{A} \otimes \sigma^{B}$.

Remark 2.8. The channel of the previous example was constructed only to emphasize that the channel need not be unital for noiseless subsystems to exist. This example is mathematically motivated, and thus it may seem somewhat artificial from the physical perspective. However, the work [18] shows that this is the typical manner in which noiseless subsystems arise for arbitrary channels. In particular, the conditions of Lemma 2.3 can be seen to be equivalent to the requirement $\mathcal{P}_{\mathfrak{A}} \circ \mathcal{E} \circ \mathcal{P}_{\mathfrak{A}}=\mathcal{E} \circ \mathcal{P}_{\mathfrak{A}}=\mathcal{E}^{\prime} \otimes i d_{B}$, where $\mathcal{E}^{\prime}$ is some channel on $\mathcal{H}^{A}$ and $i d_{B}$ is the identity channel on $\mathcal{H}^{B}$. See [18] for further discussions on this point.

\section{Operator Quantum Error Correction}

The unified scheme for quantum error correction consists of a triple $(\mathcal{R}, \mathcal{E}, \mathfrak{A})$ where again $\mathcal{R}$ and $\mathcal{E}$ are quantum operations on some $\mathcal{B}(\mathcal{H})$, but now $\mathfrak{A}$ is a semigroup in $\mathcal{B}(\mathcal{H})$ defined as above with respect to a fixed decomposition $\mathcal{H}=\left(\mathcal{H}^{A} \otimes \mathcal{H}^{B}\right) \oplus \mathcal{K}$.

Definition 3.1. Given such a triple $(\mathcal{R}, \mathcal{E}, \mathfrak{A})$ we say that the $B$-sector of $\mathfrak{A}$ is correctable for $\mathcal{E}$ if

$$
\left(\operatorname{Tr}_{A} \circ \mathcal{P}_{\mathfrak{A}} \circ \mathcal{R} \circ \mathcal{E}\right)(\sigma)=\operatorname{Tr}_{A}(\sigma) \text { for all } \sigma \in \mathfrak{A} .
$$

In other words, $(\mathcal{R}, \mathcal{E}, \mathfrak{A})$ is a correctable triple if the $\mathcal{H}^{B}$ sector of the semigroup $\mathfrak{A}$ encodes a noiseless subsystem for the error map $\mathcal{R} \circ \mathcal{E}$. Thus, substituting $\mathcal{E}$ by $\mathcal{R} \circ \mathcal{E}$ in Lemma 2.3 offers alternative equivalent definitions of a correctable triple. Since correctable codes consist of operator semigroups and algebras, we refer to this scheme as Operator Quantum Error Correction.

Observe that the standard model for error correction is given by the particular case in the OQEC model that occurs when $m=\operatorname{dim} \mathcal{H}^{A}=1$. Lemma 2.3 shows that the decoherence-free subspace and noiseless subsystem methods are captured in this model when $\mathcal{R}=\mathrm{id}$ is the identity channel and, respectively, $m=1$ and $m \geq 1$. These facts are succinctly stated in Table 1. By a "subspace" in this truth table, we mean the natural identification of a subspace $\mathcal{H}^{B}$ with the operator algebra $\mathfrak{A} \cong \mathcal{B}\left(\mathcal{H}^{B}\right)$ when $\operatorname{dim} \mathcal{H}^{A}=m=1$. Further, the term "Algebraic NS" in the table is simply meant to refer to the operator algebra subcase of the noiseless subsystem notion for arbitrary quantum operations discussed at the start of the previous section. 
Table 1: Special Cases of Operator QEC

\begin{tabular}{||c||c||}
\hline \hline $\mathfrak{A}=$ subspace & Standard QEC \\
\hline $\mathcal{R}=i d$ & Arbitrary NS \\
\hline $\mathcal{R}=i d+\mathfrak{A}=$ algebra & Algebraic NS \\
\hline $\mathcal{R}=i d+\mathfrak{A}=$ subspace & DFS \\
\hline \hline
\end{tabular}

While we focus on the general setting of operator semigroups $\mathfrak{A}$ as correctable codes, it is important to note that correctability of a given $\mathfrak{A}$ is equivalent to the precise correction of the $\dagger$-algebra $\mathfrak{A}_{0}=\mathbb{1}^{A} \otimes \mathcal{B}\left(\mathcal{H}^{B}\right)$ in the following sense.

Theorem 3.2. Let $\mathcal{E}=\left\{E_{a}\right\}$ be a quantum operation on $\mathcal{B}(\mathcal{H})$ and let $\mathfrak{A}$ be a semigroup in $\mathcal{B}(\mathcal{H})$ as above. Then the $B$-sector of $\mathfrak{A}$ is correctable for $\mathcal{E}$ if and only if there is a quantum operation $\mathcal{R}$ on $\mathcal{B}(\mathcal{H})$ such that

$$
(\mathcal{R} \circ \mathcal{E})(\sigma)=\sigma \quad \forall \sigma \in \mathfrak{A}_{0}
$$

Proof. If Eq. (14) holds, then condition 2. of Lemma 2.3 holds for $\mathcal{R} \circ \mathcal{E}$ with $\tau^{A}=\mathbb{1}^{A}$ and hence the $B$-sector of $\mathfrak{A}$ is correctable for $\mathcal{E}$. For the converse, suppose that condition 2. of Lemma 2.3 holds for $\mathcal{R} \circ \mathcal{E}$. Note that the map $\Gamma^{\prime}=\left\{\frac{1}{\sqrt{m}} P_{k l}\right\}$ is trace preserving on $\mathcal{B}\left(\mathcal{H}^{A} \otimes \mathcal{H}^{B}\right)$. Thus by Lemma 2.1 we have for all $\sigma^{B}$,

$$
\left(\Gamma^{\prime} \circ \mathcal{R} \circ \mathcal{E}\right)\left(\mathbb{1}^{A} \otimes \sigma^{B}\right)=\Gamma^{\prime}\left(\tau^{A} \otimes \sigma^{B}\right) \propto \mathbb{1}^{A} \otimes \sigma^{B} .
$$

By trace preservation the proportionality factor must be one, and hence Eq. (14) is satisfied for $\left(\Gamma^{\prime} \circ \mathcal{R}\right) \circ \mathcal{E}$. The map $\Gamma^{\prime}$ may be extended to a quantum operation on $\mathcal{B}(\mathcal{H})$ by including the projection $P_{\mathfrak{A}}^{\perp}$ onto $\mathcal{K}$ as a Kraus operator. As this does not effect the calculation Eq. (15), the result follows.

We next derive a testable condition that characterizes correctable codes for a given channel $\mathcal{E}$ in terms of its error operators and generalizes Eq. (2) for the standard model. We first glean some interesting peripheral information.

Lemma 3.3. Let $\mathcal{E}=\left\{E_{a}\right\}$ be a quantum operation on $\mathcal{B}(\mathcal{H})$ and let $P$ be a projection on $\mathcal{H}$. If $\mathcal{E}(P)=P$, then the range space $\mathcal{C}$ for $P$ is invariant for every $E_{a}$; that is,

$$
E_{a} P=P E_{a} P \quad \forall a .
$$


Proof. Let $|\psi\rangle$ belong to $\mathcal{C}=P \mathcal{H}$. Then by hypothesis and the positivity of $\mathcal{E}$, for each $a$ we have

$$
E_{a}|\psi\rangle\left\langle\psi\left|E_{a}^{\dagger} \leq \sum_{b} E_{b}\right| \psi\right\rangle\langle\psi| E_{b}^{\dagger}=\mathcal{E}(|\psi\rangle\langle\psi|) \leq \mathcal{E}(P)=P
$$

Thus $P^{\perp}\left(E_{a}|\psi\rangle\langle\psi| E_{a}^{\dagger}\right) P^{\perp} \leq P^{\perp} P P^{\perp}=0$ and so $P^{\perp} E_{a}|\psi\rangle=0$. As both $|\psi\rangle$ and $a$ were arbitrary the result follows.

An adjustment of this proof shows that more is true when $\mathcal{E}$ is contractive $(\mathcal{E}(\mathbb{1}) \leq \mathbb{1})$. Specifically, $\mathcal{E}(P) \leq P$ if and only if $E_{a} P=P E_{a} P$ for all $a$ in this event. In the special case of unital operations one can further obtain the following [17].

Proposition 3.4. If $\mathcal{E}=\left\{E_{a}\right\}$ is a unital quantum operation and $P$ is a projector, then $\mathcal{E}(P)=P$ if and only if the range space for $P$ reduces each $E_{a}$; that is, $P E_{a}=E_{a} P$ for all $a$.

We now prove necessary and sufficient conditions for a semigroup $\mathfrak{A}$ to be correctable for a given error model. Sufficiency was first proven in [19]. We assume that matrix units $\left\{P_{k l}\right\}$ inside $\mathcal{B}\left(\mathcal{H}^{A}\right) \otimes \mathbb{1}^{B}$ have been identified as above.

Theorem 3.5. Let $\mathcal{E}=\left\{E_{a}\right\}$ be a quantum operation on $\mathcal{B}(\mathcal{H})$ and let $\mathfrak{A}$ be a semigroup in $\mathcal{B}(\mathcal{H})$ as above. Then the $B$-sector of $\mathfrak{A}$ is correctable for $\mathcal{E}$ if and only if there are scalars $\Lambda=\left(\lambda_{\text {abkl }}\right)$ such that

$$
P_{k k} E_{a}^{\dagger} E_{b} P_{l l}=\lambda_{a b k l} P_{k l} \quad \forall a, b, k, l .
$$

Proof. To prove necessity, by Theorem 3.2 we can assume there is a quantum operation $\mathcal{R}$ on $\mathcal{B}(\mathcal{H})$ such that $\mathcal{R} \circ \mathcal{E}$ acts as the identity channel on $\mathfrak{A}_{0}=\mathbb{1}^{A} \otimes \mathcal{B}\left(\mathcal{H}^{B}\right) \subseteq \mathcal{B}(\mathcal{H})$. For brevity, we shall first suppose that $\mathcal{R}=$ id is the identity channel.

Let $\mathcal{C}=P_{\mathfrak{A}} \mathcal{H}$ be the range of the projection $P_{\mathfrak{A}}=P_{11}+\ldots+P_{m m}$. Then since $P_{\mathfrak{A}} \in \mathfrak{A}_{0}$ we have $\mathcal{E}\left(P_{\mathfrak{A}}\right)=P_{\mathfrak{A}}$ and so Lemma 3.3 gives us $\left.P_{\mathfrak{A}} E_{a}\right|_{\mathcal{C}}=\left.E_{a}\right|_{\mathcal{C}}$ for all $a$.

With $\mathcal{B}(\mathcal{C})$ naturally regarded as imbedded inside $\mathcal{B}(\mathcal{H})$, define a completely positive map $\mathcal{E}_{\mathcal{C}}: \mathcal{B}(\mathcal{C}) \rightarrow \mathcal{B}(\mathcal{C})$ via

$$
\sigma \mapsto \mathcal{E}_{\mathcal{C}}(\sigma)=\left.P_{\mathfrak{A}} \mathcal{E}(\sigma)\right|_{\mathcal{C}}=\left.P_{\mathfrak{A}} \mathcal{E}\left(P_{\mathfrak{A}} \sigma P_{\mathfrak{A}}\right)\right|_{\mathcal{C}}
$$

for all $\sigma \in \mathcal{B}(\mathcal{C})$. Then we have

$$
\sum_{a}\left(\left.P_{\mathfrak{A}} E_{a}\right|_{\mathcal{C}}\right)^{\dagger}\left(\left.P_{\mathfrak{A}} E_{a}\right|_{\mathcal{C}}\right)=\left.\sum_{a} P_{\mathfrak{A}} E_{a}^{\dagger} E_{a}\right|_{\mathcal{C}}=\left.P_{\mathfrak{A}} \mathbb{1}_{\mathcal{H}}\right|_{\mathcal{C}}=\mathbb{1}_{\mathcal{C}}
$$

and so $\mathcal{E}_{\mathcal{C}}$ defines a quantum operation on $\mathcal{B}(\mathcal{C})$. Moreover, $\mathcal{E}_{\mathcal{C}}$ is unital as $\mathcal{E}_{\mathcal{C}}\left(\mathbb{1}_{\mathcal{C}}\right)=\left.P_{\mathfrak{A}} \mathcal{E}\left(P_{\mathfrak{A}}\right)\right|_{\mathcal{C}}=\mathbb{1}_{\mathcal{C}}$. 
Thus by hypothesis and Eq. (5) we have

$$
\left.\mathfrak{A}_{0}\right|_{\mathcal{C}} \subseteq \operatorname{Fix}\left(\mathcal{E}_{\mathcal{C}}\right)=\left\{\left.P_{\mathfrak{A}} E_{a}\right|_{\mathcal{C}},\left.P_{\mathfrak{A}} E_{a}^{\dagger}\right|_{\mathcal{C}}\right\}^{\prime},
$$

where the latter commutant is computed inside $\mathcal{B}(\mathcal{C})$. It follows that

$$
\mathcal{B}\left(\mathcal{H}^{A}\right) \otimes \mathbb{1}^{B}=\left(\left.\mathfrak{A}_{0}\right|_{\mathcal{C}}\right)^{\prime} \supseteq\left\{\left.P_{\mathfrak{A}} E_{a}\right|_{\mathcal{C}},\left.P_{\mathfrak{A}} E_{a}^{\dagger}\right|_{\mathcal{C}}\right\}^{\prime \prime}=\mathrm{C}^{*}\left(\left\{\left.P_{\mathfrak{A}} E_{a}\right|_{\mathcal{C}}\right\}\right) .
$$

Since the $P_{k l}$ form a set of matrix units that generate $\left(\left.P_{\mathfrak{A}} \mathfrak{A}_{0}\right|_{\mathcal{C}}\right)^{\prime}=$ $\mathcal{B}\left(\mathcal{H}^{A}\right) \otimes \mathbb{1}^{B}$ as a vector space, there are scalars $\mu_{a k l} \in \mathbb{C}$ such that

$$
P_{k k} E_{a} P_{l l}=P_{k k}\left(\left.P_{\mathfrak{A}} E_{a}\right|_{\mathcal{C}}\right) P_{l l}=\mu_{a k l} P_{k l} \text {. }
$$

We now turn to the general case and suppose $\mathcal{R}=\left\{R_{b}\right\}$. The noise operators for the operation $\mathcal{R} \circ \mathcal{E}$ are $\left\{R_{b} E_{a}\right\}$ and thus we may find scalars $\mu_{a b k l}$ such that

$$
P_{k k} R_{b} E_{a} P_{l l}=\mu_{a b k l} P_{k l} \quad \forall a, b, k, l .
$$

Consider the products

$$
\begin{aligned}
\left(P_{k k} R_{b} E_{a} P_{l l}\right)^{\dagger}\left(P_{k^{\prime} k^{\prime}} R_{b} E_{a^{\prime}} P_{l^{\prime} l^{\prime}}\right) & =\left(\overline{\mu_{a b k l}} P_{l k}\right)\left(\mu_{a^{\prime} b k^{\prime} l^{\prime}} P_{k^{\prime} l^{\prime}}\right) \\
& =\left\{\begin{array}{cc}
\left(\overline{\mu_{a b k l}} \mu_{a^{\prime} b k l^{\prime}}\right) P_{l l^{\prime}} & \text { if } k=k^{\prime} \\
0 & \text { if } k \neq k^{\prime}
\end{array} .\right.
\end{aligned}
$$

Noting that $\mathcal{C}$ is invariant for the noise operators $R_{b} E_{a}$ by Lemma 3.3 . for fixed $a, a^{\prime}$ and $l, l^{\prime}$ we use $\sum_{b} R_{b}^{\dagger} R_{b}=\mathbb{1}$ to obtain

$$
\begin{aligned}
\left(\sum_{b, k} \overline{\mu_{a b k l}} \mu_{a^{\prime} b k l^{\prime}}\right) P_{l l^{\prime}} & =\sum_{b, k}\left(P_{l l} E_{a}^{\dagger} R_{b}^{\dagger} P_{k k}\right)\left(P_{k k} R_{b} E_{a^{\prime}} P_{l^{\prime} l^{\prime}}\right) \\
& =\sum_{b} P_{l l} E_{a}^{\dagger} R_{b}^{\dagger} P_{\mathfrak{A}} R_{b} E_{a^{\prime}} P_{l^{\prime} l^{\prime}} \\
& =P_{l l} E_{a}^{\dagger}\left(\sum_{b} R_{b}^{\dagger} R_{b}\right) E_{a^{\prime}} P_{l^{\prime} l^{\prime}} \\
& =P_{l l} E_{a}^{\dagger} E_{a^{\prime}} P_{l^{\prime} l^{\prime}}
\end{aligned}
$$

The proof is completed by setting $\lambda_{a a^{\prime} l l^{\prime}}=\sum_{b, k} \overline{\mu_{a b k l}} \mu_{a^{\prime} b k l^{\prime}}$ for all $a, a^{\prime}$ and $l, l^{\prime}$.

For sufficiency, let us assume that Eq. (16) holds. Let $\sigma_{k}=\left|\alpha_{k}\right\rangle\left\langle\alpha_{k}\right| \in$ $\mathcal{B}\left(\mathcal{H}^{A}\right)$, for $1 \leq k \leq m$, and define a quantum operation $\mathcal{E}_{k}: \mathcal{B}\left(\mathcal{H}^{B}\right) \rightarrow$ $\mathcal{B}(\mathcal{H})$ by $\mathcal{E}_{k}\left(\rho^{B}\right) \equiv \mathcal{E}\left(\sigma_{k} \otimes \rho^{B}\right)$. With $P \equiv P_{\mathfrak{A}}$ and $E_{a, k} \equiv E_{a} P\left|\alpha_{k}\right\rangle$, it follows that $\mathcal{E}_{k}=\left\{E_{a, k}\right\}$. We shall find a quantum operation that globally corrects all of the errors $E_{a, k}$.

To do this, first note that we may define a quantum operation $\mathcal{E}_{B}$ : $\mathcal{B}\left(\mathcal{H}^{B}\right) \rightarrow \mathcal{B}(\mathcal{H})$ with error model

$$
\mathcal{E}_{B}=\left\{\frac{1}{\sqrt{m}} E_{a, k}: \forall a, \forall 1 \leq k \leq m\right\}
$$


Then Eq. (16)) and $P=\sum_{k} P_{k k}$ give us

$$
\begin{aligned}
\mathbb{1}^{B} E_{a, k}^{\dagger} E_{b, l} \mathbb{1}^{B} & =\mathbb{1}^{B}\left\langle\alpha_{k}\left|P E_{a}^{\dagger} E_{b} P\right| \alpha_{l}\right\rangle \mathbb{1}^{B} \\
& =\sum_{k^{\prime}, l^{\prime}} \mathbb{1}^{B}\left\langle\alpha_{k}\left|P_{k^{\prime} k^{\prime}} E_{a}^{\dagger} E_{b} P_{l^{\prime} l^{\prime}}\right| \alpha_{l}\right\rangle \mathbb{1}^{B} \\
& =\sum_{k^{\prime}, l^{\prime}} \lambda_{a b k^{\prime} l^{\prime}} \mathbb{1}^{B}\left\langle\alpha_{k}\left|P_{k^{\prime} l^{\prime}}\right| \alpha_{l}\right\rangle \mathbb{1}^{B}=\lambda_{a b k l} \mathbb{1}^{B} .
\end{aligned}
$$

In particular, Standard QEC implies the existence of a quantum operation $\mathcal{R}: \mathcal{B}(\mathcal{H}) \rightarrow \mathcal{B}\left(\mathcal{H}^{B}\right)$ such that $\left(\mathcal{R} \circ \mathcal{E}_{B}\right)\left(\rho^{B}\right)=\rho^{B}$ for all $\rho^{B}$.

This implies that

$$
\begin{aligned}
(\mathcal{R} \circ \mathcal{E})\left(\mathbb{1}^{A} \otimes \rho^{B}\right) & =\mathcal{R}\left(\sum_{k} \mathcal{E}_{k}\left(\rho^{B}\right)\right) \\
& =m \mathcal{R}\left(\sum_{k, a} \frac{1}{m} E_{a, k} \rho^{B} E_{a, k}^{\dagger}\right) \\
& =m \mathcal{R} \circ \mathcal{E}_{B}\left(\rho^{B}\right)=m \rho^{B} .
\end{aligned}
$$

Hence we may define a channel $I_{\mathfrak{A}}: \mathcal{B}\left(\mathcal{H}^{B}\right) \rightarrow \mathcal{B}(\mathcal{H})$ via $I_{\mathfrak{A}}\left(\rho^{B}\right)=$ $\frac{1}{m}\left(\mathbb{1}^{A} \otimes \rho^{B}\right)$. Thus, on defining $\mathcal{R}^{\prime} \equiv I_{\mathfrak{A}} \circ \mathcal{R}$, we obtain

$$
\left(\mathcal{R}^{\prime} \circ \mathcal{E}\right)\left(\mathbb{1}^{A} \otimes \rho^{B}\right)=\mathbb{1}^{A} \otimes \rho^{B} \quad \forall \rho^{B} \in \mathcal{B}\left(\mathcal{H}^{B}\right) .
$$

The result now follows from an application of Theorem 3.2 .

Remark 3.6. The necessity of Eq. (16) for correction was initially established in [1]. Here we have provided a new operator algebra proof based on Eq. (5) and Theorem 3.2. In the original draft of this paper, we established sufficiency of Eq. (16) up to a set of technical conditions. More recently, sufficiency was established in full generality in [19]. (The work [19] also casts this condition into information theoretic language.) Here we have included an operator algebra version (based on Theorem 3.2) of the proof of sufficiency sketched in [19].

Let us note that Eq. (16) is independent of the choice of basis $\left\{\left|\alpha_{k}\right\rangle\right\}$ that define the family $P_{k l}$ and of the operator-sum representation for $\mathcal{E}$. In particular, under the changes $\left|\alpha_{k}^{\prime}\right\rangle=\sum_{l} u_{k l}\left|\alpha_{l}\right\rangle$ and $F_{a}=\sum_{b} w_{a b} E_{b}$, the scalars $\Lambda$ change to $\lambda_{a b k l}^{\prime}=\sum_{a^{\prime} b^{\prime} k^{\prime} l^{\prime}} \bar{u}_{k k^{\prime}} u_{l^{\prime} l} \bar{w}_{a a^{\prime}} w_{b b^{\prime}} \lambda_{a b k l}$.

Equation (16) generalizes the quantum error correction condition Eq. (22) to the case where information is encoded in operators, not necessarily restricted to act on a fixed code subspace $\mathcal{C}$. However, observe that setting $k=l$ in Eq. (16) gives the standard error correction condition Eq. (2) with $P_{\mathcal{C}}=P_{k k}$. This leads to the following result. 
Theorem 3.7. If $(\mathcal{R}, \mathcal{E}, \mathfrak{A})$ is a correctable triple for some semigroup $\mathfrak{A}$ defined as above, then $\left(\mathcal{P}_{k} \circ \mathcal{R}, \mathcal{E}, P_{k k} \mathfrak{A} P_{k k}\right)$ is a correctable triple according to the standard definition Eq. (2), where $P_{k k}$ is any minimal reducing projection of $\mathfrak{A}_{0}=\mathbb{1}^{A} \otimes \mathcal{B}\left(\mathcal{H}^{B}\right)$, and the map $\mathcal{P}_{k}$ is defined by $\mathcal{P}_{k}(\cdot)=\sum_{l} P_{k l}(\cdot) P_{k l}^{\dagger}$.

Proof. Let $\sigma \in\left|\alpha_{k}\right\rangle\left\langle\alpha_{k}\right| \otimes \mathcal{B}\left(\mathcal{H}^{B}\right)$, so that $\sigma=P_{k k} \sigma P_{k k}$. Let $\mathcal{E}=\left\{E_{a}\right\}$ and $\mathcal{R}=\left\{R_{b}\right\}$. By Theorem 2.5 there are scalars $\lambda_{a b k l}$ such that $P_{k k} R_{b} E_{a} P_{l l}=\lambda_{a b k l} P_{k l} \forall a, b, k, l$. It follows that

$$
\begin{aligned}
\left(\mathcal{P}_{k} \circ \mathcal{R} \circ \mathcal{E}\right)(\sigma) & =\sum_{a, b, l} P_{k l} R_{b} E_{a} P_{k k} \sigma P_{k k} E_{a}^{\dagger} R_{b}^{\dagger} P_{l k} \\
& =\sum_{a, b, l}\left(\lambda_{a b l k} P_{k k}\right) \sigma\left(\overline{\lambda_{a b l k}} P_{k k}\right) \\
& =\left(\sum_{a, b, l}\left|\lambda_{a b l k}\right|^{2}\right) \sigma .
\end{aligned}
$$

Thus $\left(\mathcal{P}_{k} \circ \mathcal{R} \circ \mathcal{E}\right)(\sigma) \propto \sigma$ for all $\sigma \in\left|\alpha_{k}\right\rangle\left\langle\alpha_{k}\right| \otimes \mathcal{B}\left(\mathcal{H}^{B}\right)$, the proportionality factor independent of $\sigma$. In fact, this factor is one. To see this, fix $k$ and note that Theorem 2.5] shows that

$$
R_{b} E_{a} P_{k k}=R_{b} E_{a} P_{\mathfrak{A}} P_{k k}=P_{\mathfrak{A}} R_{b} E_{a} P_{\mathfrak{A}} P_{k k}=P_{\mathfrak{A}} R_{b} E_{a} P_{k k} \quad \forall a, b .
$$

Hence, trace preservation of $\mathcal{R} \circ \mathcal{E}$ yields

$$
\begin{aligned}
\left(\sum_{a, b, l}\left|\lambda_{a b l k}\right|^{2}\right) P_{k k} & =\sum_{a, b, l}\left(P_{k k} E_{a}^{\dagger} R_{b}^{\dagger} P_{l l}\right)\left(P_{l l} R_{b} E_{a} P_{k k}\right) \\
& =P_{k k}\left(\sum_{a, b} E_{a}^{\dagger} R_{b}^{\dagger} P_{\mathfrak{A}} R_{b} E_{a}\right) P_{k k} \\
& =P_{k k}\left(\sum_{a, b} E_{a}^{\dagger} R_{b}^{\dagger} R_{b} E_{a}\right) P_{k k}=P_{k k}
\end{aligned}
$$

As $k$ was arbitrary, the result follows.

Remark 3.8. Theorem 3.7 has important consequences. Given a map $\mathcal{E}$, the existence of a correctable code subspace $\mathcal{C}$ - captured by the standard error correction condition Eq. (2) - is a prerequisite to the existence of any known type of error correction or prevention scheme (including the generalizations introduced here and in [1]). Moreover, Theorem 3.7 shows how to transform any one of these error correction or prevention techniques into a standard error correction scheme. However, while Operator Quantum Error Correction does not lead to new families of codes, it does allow simpler correction procedures. 
Remark 3.9. As a special case, Theorem 3.7 demonstrates that to every noiseless subsystem, there is an associated QEC code obtained by projecting the $A$-sector to a pure state. This is complementary to Theorem 6 of [10] which demonstrates that every QEC scheme composed of a triple $(\mathcal{R}, \mathcal{E}, \mathcal{C})$ arises as a noiseless subsystem of the map $\mathcal{E} \circ \mathcal{R}$.

We conclude this section by exhibiting the 2-qubit case of a new class of quantum channels, together with correctable subsystems, that is covered by OQEC but does not fit into the Standard QEC protocol. First, let us recall briefly that the motivating class of channels $\mathcal{E}=\left\{E_{a}\right\}$ which satisfy Eq. (2) occur when the restrictions $\left.E_{a}\right|_{P_{\mathcal{C}} \mathcal{H}}=\left.E_{a}\right|_{\mathcal{C}}$ of the error operators to $\mathcal{C}$ are scalar multiples of unitary operators $U_{a}$ such that the subspaces $U_{a} \mathcal{C}$ are mutually orthogonal. In fact, this case describes any error model that satisfies Eq. (2), up to a linear transformation of the error operators. In this situation the positive scalar matrix $\Lambda$ is diagonal. A correction operation here may be constructed by an application of the measurement operation determined by the subspaces $U_{a} \mathcal{C}$, followed by the reversals of the corresponding restricted unitaries $U_{a} P_{\mathcal{C}}$. Specifically, if $P_{a}$ is the projection of $\mathcal{H}$ onto $U_{a} \mathcal{C}$, then $\mathcal{R}=\left\{U_{a}^{\dagger} P_{a}\right\}$ satisfies Eq. (11) for $\mathcal{E}$ on $\mathcal{C}$. The following is a generalization of this class of channels to the OQEC setting. For clarity we focus on the 2-qubit case.

Example 3.10. Let $\left\{|a\rangle,|b\rangle,\left|a^{\prime}\right\rangle,\left|b^{\prime}\right\rangle\right\}$ and $\left\{\left|a_{1}\right\rangle,\left|b_{1}\right\rangle,\left|a_{2}\right\rangle,\left|b_{2}\right\rangle\right\}$ be two orthonormal bases for $\mathbb{C}^{4}$. Let $P_{1}$ be the projection onto $\operatorname{span}\{|a\rangle,|b\rangle\}$ and $P_{2}$ the projection onto $\operatorname{span}\left\{\left|a^{\prime}\right\rangle,\left|b^{\prime}\right\rangle\right\}$. Let $Q_{i}, i=1,2$, be the projection onto $\operatorname{span}\left\{\left|a_{i}\right\rangle,\left|b_{i}\right\rangle\right\}$. Define partial isometries (i.e., unitary operators restricted to a subspace of the full system space) $U_{1}=U_{1} P_{1}$, $U_{1}^{\prime}=U_{1}^{\prime} P_{2}, U_{2}=U_{2} P_{1}, U_{2}^{\prime}=U_{2}^{\prime} P_{2}$ on $\mathbb{C}^{4}$ by

$$
\left\{\begin{array} { r l } 
{ U _ { 1 } | a \rangle } & { = | a _ { 1 } \rangle } \\
{ U _ { 1 } | b \rangle } & { = | b _ { 1 } \rangle } \\
{ U _ { 1 } ^ { \prime } | a ^ { \prime } \rangle } & { = | a _ { 1 } \rangle } \\
{ U _ { 1 } ^ { \prime } | b ^ { \prime } \rangle } & { = | b _ { 1 } \rangle }
\end{array} \quad \left\{\begin{array}{rl}
U_{2}|a\rangle & =\left|a_{2}\right\rangle \\
U_{2}|b\rangle & =\left|b_{2}\right\rangle \\
U_{2}^{\prime}\left|a^{\prime}\right\rangle & =\left|a_{2}\right\rangle \\
U_{2}^{\prime}\left|b^{\prime}\right\rangle & =\left|b_{2}\right\rangle
\end{array} .\right.\right.
$$

Then the operators $\mathcal{E}=\left\{E_{1}, E_{2}\right\}$ define a quantum channel where

$$
\begin{aligned}
& E_{1}=\frac{1}{\sqrt{2}}\left(U_{1} P_{1}+U_{1}^{\prime} P_{2}\right) \\
& E_{2}=\frac{1}{\sqrt{2}}\left(U_{2} P_{1}-U_{2}^{\prime} P_{2}\right) .
\end{aligned}
$$

The action of $E_{1}$ and $E_{2}$ is indicated in Figure 1. 
FiguRe 1.

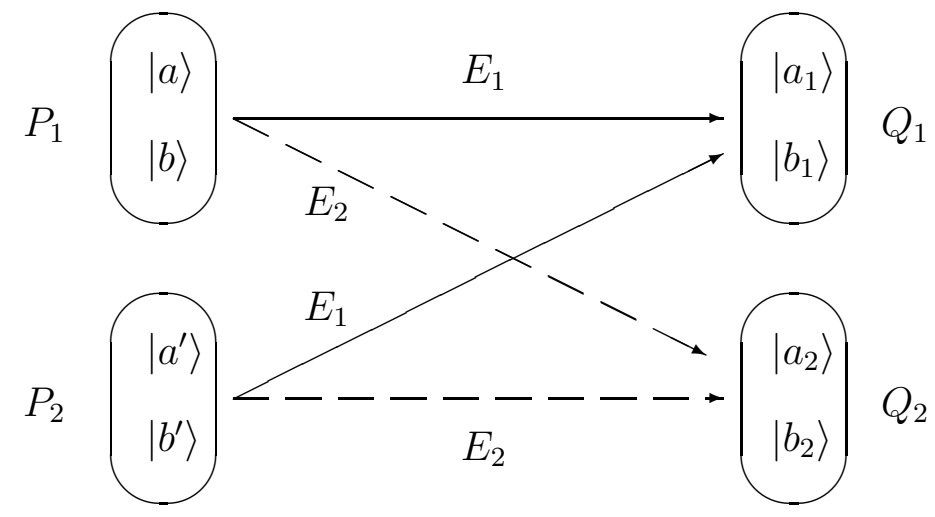

Here the matrix units are given by

$$
\begin{gathered}
P_{1}=P_{11}=|a\rangle\langle a|+| b\rangle\langle b| \\
P_{2}=P_{22}=\left|a^{\prime}\right\rangle\left\langle a^{\prime}|+| b^{\prime}\right\rangle\left\langle b^{\prime}\right| \\
P_{12}=|a\rangle\left\langle a^{\prime}|+| b\right\rangle\left\langle b^{\prime}\right| \\
P_{21}=\left|a^{\prime}\right\rangle\left\langle a|+| b^{\prime}\right\rangle\langle b| .
\end{gathered}
$$

For trace preservation, observe that

$$
\begin{aligned}
E_{1}^{\dagger} E_{1} & =\frac{1}{2}\left(P_{1} U_{1}^{\dagger}+P_{2}\left(U_{1}^{\prime}\right)^{\dagger}\right)\left(U_{1} P_{1}+U_{1}^{\prime} P_{2}\right) \\
& =\frac{1}{2}\left(P_{11}+P_{12}+P_{21}+P_{22}\right) .
\end{aligned}
$$

Similarly, we compute

$$
E_{2}^{\dagger} E_{2}=\frac{1}{2}\left(P_{11}-P_{12}-P_{21}+P_{22}\right) .
$$

Thus we have $E_{1}^{\dagger} E_{1}+E_{2}^{\dagger} E_{2}=P_{11}+P_{22}=\mathbb{1}_{4}$. Equations (16) are computed as follows:

$$
\begin{gathered}
P_{k} E_{i}^{\dagger} E_{i} P_{k}=\frac{1}{2} P_{k} \text { for } i, k=1,2, \\
P_{k} E_{i}^{\dagger} E_{j} P_{l}=0 \text { for } i \neq j \text { and } k, l=1,2, \\
P_{1} E_{1}^{\dagger} E_{1} P_{2}=\frac{1}{2} P_{12}=\left(\frac{1}{2} P_{21}\right)^{\dagger}=\left(P_{2} E_{1}^{\dagger} E_{1} P_{1}\right)^{\dagger}, \\
P_{1} E_{2}^{\dagger} E_{2} P_{2}=\frac{-1}{2} P_{12}=\left(\frac{-1}{2} P_{21}\right)^{\dagger}=\left(P_{2} E_{2}^{\dagger} E_{2} P_{1}\right)^{\dagger} .
\end{gathered}
$$

Define

$$
V_{11}=U_{1} P_{1}, \quad V_{12}=U_{1}^{\prime} P_{2}, \quad V_{21}=U_{2} P_{1}, \quad V_{22}=U_{2}^{\prime} P_{2}
$$


and observe that

$$
\begin{aligned}
& V_{11} V_{11}^{\dagger}=U_{1} P_{1} U_{1}^{\dagger}=Q_{1}=U_{1}^{\prime} P_{2}\left(U_{1}^{\prime}\right)^{\dagger}=V_{12} V_{12}^{\dagger} \\
& V_{21} V_{21}^{\dagger}=U_{2} P_{1} U_{2}^{\dagger}=Q_{2}=U_{2}^{\prime} P_{2}\left(U_{2}^{\prime}\right)^{\dagger}=V_{22} V_{22}^{\dagger} .
\end{aligned}
$$

Then a calculation shows that the channel

$$
\mathcal{R}=\left\{\frac{1}{\sqrt{2}} V_{j k}^{\dagger} Q_{j}: 1 \leq j, k \leq 2\right\}
$$

corrects for all errors induced by $\mathcal{E}$ on $\mathfrak{A}_{0} \cong \mathbb{1}_{2} \otimes \mathcal{M}_{2}$. Specifically, $(\mathcal{R} \circ \mathcal{E})(\sigma)=\sigma$ for all $\sigma \in \mathcal{B}\left(\mathbb{C}^{4}\right)$ which have a matrix representation of the form $\sigma=\left(\begin{array}{cc}\sigma_{1} & 0 \\ 0 & \sigma_{1}\end{array}\right), \sigma_{1} \in \mathcal{M}_{2}$, with respect to the ordered basis $\left\{|a\rangle,|b\rangle,\left|a^{\prime}\right\rangle,\left|b^{\prime}\right\rangle\right\}$ for $\mathbb{C}^{4}$. That is, $(\mathcal{R} \circ \mathcal{E})(\sigma)=\sigma$ for all $\alpha_{11}, \alpha_{12}, \alpha_{21}, \alpha_{22} \in \mathbb{C}$ and all

$$
\begin{aligned}
\sigma= & \alpha_{11}\left(|a\rangle\left\langle a|+| a^{\prime}\right\rangle\left\langle a^{\prime}\right|\right)+\alpha_{12}\left(|a\rangle\left\langle b|+| a^{\prime}\right\rangle\left\langle b^{\prime}\right|\right) \\
& +\alpha_{21}\left(|b\rangle\left\langle a|+| b^{\prime}\right\rangle\left\langle a^{\prime}\right|\right)+\alpha_{22}\left(|b\rangle\left\langle b|+| b^{\prime}\right\rangle\left\langle b^{\prime}\right|\right) .
\end{aligned}
$$

Thus $\mathcal{R}$ corrects all $\sigma=\mathbb{1}_{2} \otimes \sigma_{1}$ that are "equally balanced" with respect to the standard bases for the ranges of $P_{1}$ and $P_{2}$. Further, by Theorem 3.2 we know $\mathcal{R}$ corrects the associated semigroup $\mathfrak{A}$ in the sense of Definition 3.1 .

Remark 3.11. We note that recent work [20] presents physically motivated examples in which correction of subsystems is accomplished within the Operator QEC framework. Furthermore, a general class of recovery procedures based on the stabilizer formalism was recently presented in [21]. In particular, this work uses the Operator QEC theory to present a modified version of Shor's 9-qubit code where a non-trivial "noisy subsystem" is identified and leads to a simplification of the error correction procedure and an extension of the class of logical operations.

\section{Unitarily Noiseless Subsystems}

In this section we discuss error triples $(\mathcal{R}, \mathcal{E}, \mathfrak{A})$ such that the restriction of $\mathcal{R}$ to $\mathcal{E}(\mathfrak{A})$ is a unitary operation. Consideration of this case leads to a generalization of the noiseless subsystem protocol that falls under the OQEC umbrella. Let us first consider a direct generalization of the fixed point set algebraic approach as in Eq. (15). Here we have the equation

$$
\mathcal{E}(\sigma)=U \sigma U^{\dagger} \quad \forall \sigma \in \mathfrak{A}_{0}=\mathbb{1}^{A} \otimes \mathcal{B}\left(\mathcal{H}^{B}\right),
$$

for some unitary operator $U$. When $\mathfrak{A}_{0}$ satisfies Eq. (17) for a unitary $U$ we shall say that $\mathfrak{A}_{0}$ is a unitarily noiseless subsystem (UNS) for $\mathcal{E}$. Of course, a subsystem $\mathfrak{A}_{0}$ that satisfies Eq. (17) is not noiseless, but it may be easily corrected by applying the reversal operation $U^{\dagger}(\cdot) U$. As we 
indicate below, this can lead to new non-trivial correctable subsystems not obtained under the noiseless subsystem regime. If $\mathcal{E}$ is a unital operation, it is possible to explicitly compute all UNS's for $\mathcal{E}$.

Theorem 4.1. If $\mathcal{E}=\left\{E_{a}\right\}$ is a unital quantum operation on $\mathcal{B}(\mathcal{H})$ and $U$ is a unitary on $\mathcal{H}$, then the corresponding unitarily noiseless subsystem $\mathfrak{A}_{0}$ is equal to the commutant of the operators $\left\{U^{\dagger} E_{a}\right\}$; that $i s$,

$$
\begin{aligned}
\mathfrak{A}_{0} & =\left\{\sigma \in \mathcal{B}(\mathcal{H}): \mathcal{E}(\sigma)=\sum_{a} E_{a} \sigma E_{a}^{\dagger}=U \sigma U^{\dagger}\right\} \\
& =\left\{U^{\dagger} E_{a}\right\}^{\prime} .
\end{aligned}
$$

Proof. The set of $\sigma$ that satisfy Eq. (17) is equal to the set of $\sigma$ that satisfy $U^{\dagger} \mathcal{E}(\sigma) U=\sigma$. Thus, here we are considering the fixed point set for the unital operation $U^{\dagger} \mathcal{E}(\cdot) U$, which has noise operators $\left\{U^{\dagger} E_{a}\right\}$. The result now follows from Eq. (5).

Let us consider a simple example of how this scheme can be used to identify new correctable codes for a given channel.

Example 4.2. Let $Z_{1}=Z \otimes \mathbb{1}_{2}$ and $Z_{2}=\mathbb{1}_{2} \otimes Z$ with the Pauli matrix $Z=\left(\begin{array}{cc}1 & 0 \\ 0 & -1\end{array}\right)$. Then, with respect to the standard orthonormal basis $\{|00\rangle,|01\rangle,|10\rangle,|11\rangle\}$ for $\mathbb{C}^{4}$, we have

$$
\left\{Z_{1}, Z_{2}\right\}^{\prime}=\left\{\left(\begin{array}{cccc}
a & 0 & 0 & 0 \\
0 & b & 0 & 0 \\
0 & 0 & c & 0 \\
0 & 0 & 0 & d
\end{array}\right): a, b, c, d \in \mathbb{C}\right\}
$$

Hence there are no non-trivial noiseless subsystems for the corresponding channel $\mathcal{E}=\left\{Z_{1}, Z_{2}\right\}$. However, if we let $U \in \mathcal{B}\left(\mathbb{C}^{4}\right)$ be the unitary

$$
U|i j\rangle=\left\{\begin{array}{cl}
|i j\rangle & \text { if } i \neq 1 \text { or } j \neq 1 \\
-|11\rangle & \text { if } i=1 \text { and } j=1
\end{array},\right.
$$

then we compute

$$
\left\{U^{\dagger} Z_{1}, U^{\dagger} Z_{2}\right\}^{\prime}=\left\{\left(\begin{array}{cccc}
a & 0 & 0 & b \\
0 & c & 0 & 0 \\
0 & 0 & d & 0 \\
e & 0 & 0 & f
\end{array}\right): a, b, c, d, e, f \in \mathbb{C}\right\} .
$$

In particular, the $\dagger$-algebra $\mathfrak{A}_{0}=\left\{U^{\dagger} Z_{i}\right\}^{\prime}$ is unitarily equivalent to $\mathfrak{A}_{0} \cong \mathcal{M}_{2} \oplus \mathbb{C} \oplus \mathbb{C}$. Thus, a single qubit code subspace may be corrected. Specifically, all operators $\sigma \in \mathfrak{A}_{0}$ may be corrected by applying $U^{\dagger}(\cdot) U$ since they satisfy $\mathcal{E}(\sigma)=U \sigma U^{\dagger}$. 
In a similar manner we can extend this discussion to the case of noiseless subsystems for arbitrary quantum operations. The analogue of Eq. (17) in this case is

$$
\forall \sigma^{A} \forall \sigma^{B}, \exists \tau^{A}: \mathcal{E}\left(\sigma^{A} \otimes \sigma^{B}\right)=U\left(\tau^{A} \otimes \sigma^{B}\right) U^{\dagger},
$$

where $U$ is a fixed unitary on $\mathcal{H}$. In effect, this is the special case of the OQEC formulation Eq. (13) where the recovery $\mathcal{R}$ is unitary. In this context the conditions of Lemma 2.3 yield the following.

Theorem 4.3. Given a fixed decomposition $\mathcal{H}=\left(\mathcal{H}^{A} \otimes \mathcal{H}^{B}\right) \oplus \mathcal{K}, a$ map $\mathcal{E}$ on $\mathcal{B}(\mathcal{H})$ and a unitary $U$ on $\mathcal{H}$, the following three conditions are equivalent:

(1) Eq. (18) is satisfied.

(2) $\forall \sigma^{B}, \exists \tau^{A}: \mathcal{E}\left(\mathbb{1}^{A} \otimes \sigma^{B}\right)=U\left(\tau^{A} \otimes \sigma^{B}\right) U^{\dagger}$.

(3) $\forall \sigma \in \mathfrak{A}:\left(\operatorname{Tr}_{A} \circ \mathcal{P}_{\mathfrak{A}} \circ \mathcal{U}^{-1} \circ \mathcal{E}\right)(\sigma)=\operatorname{Tr}_{A}(\sigma)$.

where $\mathcal{U}^{-1}(\cdot)=U^{\dagger}(\cdot) U$.

After our initial draft was posted on ArXiv.org, the paper [13] was posted that presents a theoretical framework which attempts to unify the full dynamics of quantum computation. The key condition in [13], called the "Quantum Computer Condition" (QCC), is motivated by a particular formula (see Eq. (19) below). It turns out that this formula is captured as a special case of UNS, and hence of OQEC.

To see this, first note that Eq. (8) from [13] is given by

$$
\mathcal{M}_{\text {dec }}\left(P \cdot\left(\mathcal{M}_{\text {enc }}(\sigma)\right)\right)=U \sigma U^{\dagger} .
$$

Here, the operators $\sigma$ act on a Hilbert space $\mathcal{H}_{\text {logical }}$, that has dimension no larger than a Hilbert space $\mathcal{H}_{\text {comp }}$, on which $P$ is a quantum operation. The encoding and decoding superoperators satisfy $\mathcal{M}_{\text {enc }}: \mathcal{B}\left(\mathcal{H}_{\text {logical }}\right) \rightarrow \mathcal{B}\left(\mathcal{H}_{\text {comp }}\right)$ and $\mathcal{M}_{\text {dec }}: \mathcal{B}\left(\mathcal{H}_{\text {comp }}\right) \rightarrow \mathcal{B}\left(\mathcal{H}_{\text {logical }}\right)$.

On the one hand, notice that if we are given a formulation as in Eq. (19) for some unitary $U_{0}$ on $\mathcal{H}_{\text {logical }}$, then since $\operatorname{dim} \mathcal{H}_{\text {logical }} \leq$ $\operatorname{dim} \mathcal{H}_{\text {comp }}$, we may identify $\mathcal{H}_{\text {logical }}$ with a subspace of $\mathcal{H}_{\text {comp }}$ via an intertwining unitary $V$ from $\mathcal{H}_{\text {logical }}$ into $\mathcal{H}_{\text {comp }}$. (We shall identify $\mathcal{H}_{\text {logical }}$ with $V\left(\mathcal{H}_{\text {logical }}\right)$.) Hence, $\mathcal{H}_{\text {comp }}=\left(\mathcal{H}_{1} \otimes \mathcal{H}_{\text {logical }}\right) \oplus \mathcal{K}$, where $\mathcal{K}=\left(\mathcal{H}_{1} \otimes \mathcal{H}_{\text {logical }}\right)^{\perp}$ and $\mathcal{H}_{1}=\mathbb{C}$ is one-dimensional. Then Eq. (19) translates to a special case of Eq. (18) with the substitution $\mathcal{E}=$ $\mathcal{M}_{\text {dec }} \circ P \circ\left(\mathcal{M}_{\text {enc }} \oplus \mathrm{id}_{\mathcal{K}}\right)$, and the unitary $U$ given by $U=1 \otimes U_{0}$.

On the other hand, suppose we have a system Hilbert space $\mathcal{H}=$ $\left(\mathcal{H}^{A} \otimes \mathcal{H}^{B}\right) \oplus \mathcal{K}$, a quantum operation $\mathcal{E}$ and unitary $U$ of the form $U=\operatorname{id}_{A} \otimes U_{0}$ where $U_{0}$ is a unitary on $\mathcal{H}^{B}$, that satisfy Eq. (18). Then by Theorem 4.3, Eq. (18) may be rewritten in the form of Eq. (19) with 
the substitutions $\mathcal{H}_{\text {logical }}=\mathcal{H}^{B}$ and $\mathcal{H}_{\text {comp }}=\mathcal{H}$. The maps are given by $P=\mathcal{E}, \mathcal{M}_{\text {enc }}\left(\sigma^{B}\right)=\mathbb{1}^{A} \otimes \sigma^{B}$ and $\mathcal{M}_{\mathrm{dec}}=\operatorname{Tr}_{A} \circ \mathcal{P}_{\mathfrak{A}}$.

\section{Conclusion}

We have presented a detailed analysis of the OQEC protocol for error correction in quantum computing. This approach provides a unified framework for investigations into both active and passive error correction techniques. Fundamentally, we have generalized the setting for correction from states to operators. The condition from standard quantum error correction was shown to be necessary for any of these schemes to be feasible. Included in this protocol is a scheme for identifying noiseless subsystems that applies to arbitrary (not necessarily unital) quantum operations. We introduced the notion of unitarily noiseless subsystems as a natural relaxation of the noiseless subsystem condition. In the updated draft of this paper, we have shown that this coincides with the central notion in the formulation of the quantum computer condition from [13].

Acknowledgements. We thank Man-Duen Choi, Michael Nielsen, Harold Ollivier, Rob Spekkens and our other colleagues for helpful discussions. This work was supported in part by funding from NSERC, CIAR, MITACS, NATEQ, and ARDA.

\section{REFERENCES}

[1] D. W. Kribs, R. Laflamme, and D. Poulin, Phys. Rev. Lett. 94, 180501 (2005).

[2] P. W. Shor, Phys. Rev. A 52, R2493 (1995).

[3] A. M. Steane, Phys. Rev. Lett. 77, 793 (1996).

[4] C. H. Bennett, D. P. DiVincenzo, J. A. Smolin, and W. K. Wootters, Phys. Rev. A 54, 3824 (1996).

[5] E. Knill and R. Laflamme, Phys. Rev. A 55, 900 (1997).

[6] G.M. Palma, K.-A. Suominen and A. Ekert, Proc. Royal Soc. A 452, 567 (1996).

[7] L.-M. Duan and G.-C. Guo, Phys. Rev. Lett. 79, 1953 (1997).

[8] P. Zanardi and M. Rasetti, Phys. Rev. Lett. 79, 3306 (1997).

[9] D.A. Lidar, I.L. Chuang, and K.B. Whaley, Phys. Rev. Lett. 81, 2594 (1998).

[10] E. Knill, R. Laflamme, and L. Viola, Phys. Rev. Lett. 84, 2525 (2000).

[11] P. Zanardi, Phys. Rev. A 63, 12301 (2001).

[12] J. Kempe, D. Bacon, D. A. Lidar, and K. B. Whaley, Phys. Rev. A 63, 42307 (2001).

[13] G. Gilbert, M. Hamrick, and F. J. Thayer, arxiv.org/quant-ph/0507141.

[14] W. Arveson, Springer - Verlag, New York - Heidelberg, 1976.

[15] K. R. Davidson, Amer. Math. Soc., Providence, 1996.

[16] M. Takesaki, Springer - Verlag, New York - Heidelberg, 1979.

[17] D. W. Kribs, Proc. Edin. Math. Soc. 46 (2003).

[18] M. D. Choi and D. W. Kribs, arxiv.org/quant-ph/0507213. 
[19] M. A. Nielsen and D. Poulin, arxiv.org/quant-ph/0506069.

[20] D. Bacon, arxiv.org/quant-ph/0506023.

[21] D. Poulin, arxiv.org/quant-ph/0508131.

${ }^{1}$ Department of Mathematics and Statistics, University of Guelph, Guelph, Ontario, Canada N1G 2W1

${ }^{2}$ Institute for Quantum Computing, University of Waterloo, WaTERLOO, ON, CANADA N2L 3G1

${ }^{3}$ Perimeter Institute for Theoretical Physics, 31 Caroline St. North, WATERLOO, ON, CANADA N2L 2Y5

${ }^{4}$ School of Physical Sciences, The University of Queensland, QLD 4072, Australia 